https://doi.org/10.15407/ujpe64.10.886

A.N. SLAVIN

Department of Physics, Oakland University

(Rochester, MI 48309, USA; e-mail: slavin@oakland.edu)

\title{
INTRODUCTION TO THE JUBILEE ISSUE OF THE UKRAINIAN JOURNAL OF PHYSICS DEVOTED TO THE 80TH BIRTHDAY OF PROFESSOR GENNADII MELKOV
}

\section{Introduction}

This issue of the Journal is devoted to the 80th jubilee of Professor Gennadii A. Melkov - one of the most internationally recognized and respected Ukrainian scientists working in the field of radiophysics and physics of magnetic and superconducting materials.

Professor G.A. Melkov is an outstanding scientist and one of the world's leading specialists in the field of microwave properties of solids. In particular, Professor G.A. Melkov discovered and investigated in detail the following important new physical effects in microwave ferrites:

- Multiquantum microwave absorption and radiation;

- Kinetic instability of microwave spin waves;

- Fine structure of nonlinear microwave susceptibility;

- Size effects in parametric excitation of magnons;

- Wave front reversal of microwave spin waves in magnetic films;

- Bose-Einstein condensation of magnons at room temperature;

- Nonlinear dynamics of magnons in metallic magnetic films.

The pioneering experimental work of Professor G.A. Melkov created a basis for our current understanding of the process of development of the spin wave turbulence in magnetic crystals above the threshold of their parametric excitation. His seminal re-

(C) A.N. SLAVIN, 2019 search of the linear and nonlinear properties of magnetodynamic resonance provided a foundation for the developments of a new type of ferrite frequency multipliers.

Professor G.A. Melkov is one of the founding fathers of a new field of modern solid state electronics - spin wave functional electronics and microwave spintronics. His fundamental results in experimental and theoretical investigations of the frequency conversion and the parametric amplification of magnetostatic waves are among the most brilliant and important in this rapidly growing field. He developed a completely new and highly efficient microwave signal processing device: multi-functional parametric signal processor that combines the functions of an active and tunable delay line, microwave amplifier, convolver, generator of a reversed phase-conjugated wave with inverted time profile, and a compressor of duration of microwave radio-pulses.

The outstanding contributions of Professor G.A. Melkov to the physics of microwave ferrites are well known and widely recognized all over the world. In 1995, he was invited to present a special lecture on the effect of kinetic instability of spin waves (discovered and investigated by him) at the most prestigious international seminar on nonlinear dynamics of spin waves in Rome, where other invited lecturers were such classics of this field as H. Suhl, E. Schloemann, and C. Patton.

The book "Magnetization Oscillations and Waves" written by Professor G.A. Melkov in collaboration with Professor A.G. Gurevich and published by CRC

ISSN 2071-0194. Ukr. J. Phys. 2019. Vol. 64, No. 10 
Publishing House is considered to be one of the best reference books on this subject in the international scientific literature.

In 2006, Professor G.A. Melkov in collaboration with the research groups of Professor S. Demokritov from Münster, Germany and Professor B. Hillebrands from Kaiserslautern, Germany experimentally demonstrated the Bose-Einstein condensation of magnons at room temperature taking place under the parametric pumping in ferrite films. The research paper on that subject was published in the "Nature" in 2006 (see Nature 443, 430 (2006)) and was named as one of the best research papers in physics in 2006 (see link http://physicsweb.org/articles/news/10/ $12 / 15 / 1$ to the see September 2006 section of "physicsweb" showing the list of twelve papers named "the best" in physics in 2006). In a recent paper by Professor G.A. Melkov and his collaborators, a qualitative explanation was provided on why the Bose-Einshein condensation of magnons is possible at room temperature (see Nature Commun. 5, 3452 (2014)).

A high international level of Professor G.A. Melkov's experimental research is demonstrated by a series of his publications in one of the most prestigious scientific journal in Physics: Physical Review Letters - see Phys. Rev. Lett. 79, 2137 (1997), Phys. Rev. Lett. 84, 3438 (2000), Phys. Rev. Lett. 86, 4918 (2001), Phys. Rev. Lett. 99, 227202 (2007), Phys. Rev. Lett. 99, 037205 (2007), Phys. Rev. Lett. 100, 047205 (2008). In these papers, Professor G.A. Melkov reported discoveries of several new and important nonlinear effects: the efficient parametric amplification of spin wave envelope solitons in ferrite garnet films by a localized parallel pumping, reversal of a wave front, and inversion of the time profile of a microwave pulse in a first-order three-wave process of parametric interaction, reversal of two-magnon momentum relaxation by a frequency selective parametric amplification, and spontaneous coherence of the Bose-Einstein condensate of magnons.

Recent scientific achievements of Professor G.A. Melkov published in the leading international scientific journals are equally impressive. He studied such diverse effects as noise in resonance-type spin-torque microwave detectors (Appl. Phys. Lett. 99, 032507 (2011)), collective spin-wave excitations in a two-dimensional array of coupled magnetic nanodots (Phys. Rev. B 85, 014427 (2012)), properties of nano-structured magnetic metamaterials with enhanced nonlinear properties (Sci. Rep. 2, 478 (2012)), conditions for the spin wave nonreciprocity in an array of dipolarly coupled magnetic nanopillars (Appl. Phys. Lett. 103, 082407 (2013)), and detection of spin waves in permalloy using the planar Hall effect (Phys. Rev. Applied 4, 014014 (2015)).

Professor G.A. Melkov continues to be one of the leading experimental researchers in the rapidly developing field of microwave magnetization dynamics, which is reflected in frequent invitations to present his latest research findings that he receives from the largest and the most prestigious international conferences on Magnetism and Nonlinear Waves such as: "Nonlinear Waves - Theory and Applications", Beijing, China, June 2008, "INTERMAG-09", Sacramento, California, USA, May 2009, "MAGNONICS09", Dresden, Germany, August 2009.

One of the most significant contributions of Professor G.A. Melkov to the field of magnetization dynamics and microwave signal processing is the creation of a scientific school of Gennadii Melkov. His former students became real scientific leaders and are successfully working in the best scientific centers and universities all over the world. For example, Alexander Serga and Vitaliy Vasyuchka are the leading researchers at the University of Kaiserlautern, Germany, Vasyl Tyberkevych is a professor at the Oakland University, USA, Andrii Chumak is a professor at the University of Vienna, Austria, Dmytro Bozhko has recently became a professor at the University of Colorado - Colorado Springs, USA, Oleksandr Dzyapko is working at the University of Münster, Germany, and Oleksandr Prokopenko is a professor and the head of a chair at Taras Shevchenko National University of Kyiv.

I would like to sincerely congratulate Professor G.A. Melkov on his anniversary, and also to congratulate the Faculty of Radiophysics, Electronics and Computer Systems of Taras Shevchenko National University of Kyiv on having such a distinguished and internationally recognized scientist as Professor G.A. Melkov.

Received 22.01.09 